\title{
Serine 389 phosphorylation of 3-phosphoinositide-dependent kinase 1 by UNC-51-like kinase 1 affects its ability to regulate Akt and p70 S6kinase
}

\author{
Kidae Kim ${ }^{1,2}$, Sung Goo Park ${ }^{1,3}$, Byoung Chul Park ${ }^{1,2 *}$, Jeong-Hoon Kim ${ }^{1,3^{*}}$ E Sunhong Kim ${ }^{1,4 *}$ \\ ${ }^{1}$ Disease Target Structure Research Center, Korea Research Institute of Bioscience and Biotechnology, Daejeon 34141, ${ }^{2}$ Department of \\ Proteome Structural biology, KRIBB School of Bioscience, Korea University of Science and Technology, Daejeon 34113 , ${ }^{3}$ Department of \\ Functional Genomics, KRIBB School of Bioscience, Korea University of Science and Technology, Daejeon 34113, ${ }^{4}$ Department of \\ Bio-Molecular Science, KRIBB School of Bioscience, Korea University of Science and Technology, Daejeon 34113, Korea
}

\begin{abstract}
Phosphorylation of the signaling component by protein kinase often leads to a kinase cascade or feedback loop. 3-Phosphoinositide-dependent kinase 1 (PDK1) signaling pathway diverges into various kinases including Akt and p70 S6 kinase (p70S6k). However, the PDK1 feedback mechanism remains elusive. Here, we demonstrated that UNC-51-like kinase (ULK1), an autophagy initiator kinase downstream of mechanistic target of rapamycin (mTOR), directly phosphorylated PDK1 on serine 389 at the linker region. Furthermore, our data showed that this phosphorylation affected the kinase activity of PDK1 toward downstream substrates. These results suggest a possible negative feedback loop between PDK1 and ULK1. [BMB Reports 2020; 53(7): 373-378]
\end{abstract}

\section{INTRODUCTION}

Altered phosphorylation induces conformational changes in proteins and modifies their function. Phosphorylation events can be considered as vehicles of intracellular communication. Protein kinases mediating the phosphorylation are also regulated via post-translational addition of phosphate groups. This phenomenon occurs repeatedly in a kinase cascade or a feedback circuit.

The phosphatidylinositol 3-kinase (PI3K)-dependent signaling pathway has long been recognized as a key regulatory me-

*Corresponding authors. Byoung Chul Park, Tel: +82-42-860-4260; Fax: +82-42-879-8596; E-mail: parkbc@kribb.re.kr; Jeong-Hoon Kim, Tel: +82-42-860-4264; Fax: +82-42-879-8596; E-mail: jhoonkim@ kribb.re.kr; Sunhong Kim, Tel: +82-42-860-4278; Fax: +82-42-8798596; E-mail: sunhong@kribb.re.kr

https://doi.org/10.5483/BMBRep.2020.53.7.299

Received 9 December 2019, Revised 16 December 2019, Accepted 19 December 2019

Keywords: Feedback loop, Linker, PDK1, Phosphorylation, ULK1 chanism in several important biological functions, such as cell proliferation, survival, and immunity (1). PI3K increases the level of phosphatidylinositol 3,4,5-trisphosphate $\left(\mathrm{PIP}_{3}\right)$ and phosphatidylinositol 3, 4-bisphosphate $\left(\mathrm{PIP}_{2}\right)$, which elicit membrane translocation of PDK1 and Akt via their pleckstrin homology $(\mathrm{PH})$ domain (2), and in turn, the phosphorylation and activation of Akt by PDK1 in a PIP $_{3}$ - or PIP $_{2}$-dependent manner $(3,4)$. Activation of PDK1 also leads to phosphorylation of p70S6k (5), serum/glucocorticoid regulated kinase (SGK) (6), p90 ribosomal protein S6 kinase (p90RSK) (7) and members of protein kinase $C$ (PKC) family, all of which belong to AGC kinase family named after protein kinase $A$, protein kinase $G$, and $\operatorname{PKC}(8,9)$.

Since PDK1 is considered constitutively active, the activity of PDK1 was measured by the degree of substrate protein modification (10). However, several studies revealed that various molecular switches may regulate PDK1 kinase activity. For example, phosphorylation at the activation loop is essential for its activity (11). Additionally, the localization of PDK1 is one of the factors that affect its function (12-14). Homodimerization of PDK1 may represent an inactive state of the kinase $(15,16)$.

ULK1 is a protein kinase that is well conserved from budding yeast to humans and is one of the components of the initiating complex downstream of the mTOR in autophagy pathway (17-20). The mTOR phosphorylates and inhibits ULK1 complex to suppress autophagy $(17,21)$, while ULK1 regulates mTOR/p70S6k signaling via phosphorylation of AMPK, mTOR, and raptor (22-25). This feedback loop between mTOR and ULK1 is important to prevent excessive autophagy. Other upstream components of mTOR signaling pathway may be regulated by ULK1 as a negative or positive feedback.

In the present study, we showed that ULK1 phosphorylated Ser389 of PDK1 at the linker region. This phosphorylation did not affect the kinase activity of PDK1. However, the nonphosphorylatable mutant of PDK1 showed weak activity toward the downstream kinases, such as Akt and p70S6k. Our data suggest a possible feedback loop between ULK1 and PDK1.

ISSN: 1976-670X (electronic edition)

Copyright (c) 2020 by the The Korean Society for Biochemistry and Molecular Biology

(c) This is an open-access article distributed under the terms of the Creative Commons Attribution Non-Commercial License (http://creativecommons.org/licenses/by-nc/4.0) which permits unrestricted non-commercial use, distribution, and reproduction in any medium, provided the original work is properly cited. 


\section{RESULTS}

\section{ULK1 interacts with PDK1 and induces phosphorylation}

All known substrates of PDK1 are located in the activation loop of AGC kinase family (26). Since the activation loop of ULK1 is very similar to the PDK1 substrate consensus sequence, we thought that PDK1 may regulate ULK1, and therefore, we evaluated the band shift of each protein by western blot analysis. Interestingly, the overexpression of HA-ULK1 increases the upper band of overexpressed and endogenous PDK1 (Fig. 1A and 1B). To confirm that this increase is related to the phosphorylation, we used ULK1 kinase inactive mutant (ULK1 KI) and $\lambda$-phosphatase. ULK1 KI failed to induce slower migrating bands of PDK1 and the $\lambda$-phosphatase treatment reduced the up-shifted bands of PDK1 by ULK1 WT (Fig. 1C), indicating that ULK1 induced the phosphorylation of PDK1. To examine the direct interaction between PDK1 and ULK1, exogenously expressed GST-PDK1 and HA-ULK1 were analyzed via co-immunoprecipitation. We found that GST-PDK1 strongly binds to HA-ULK1 (Fig. 1D). To identify the domains of PDK1 interacting with ULK1, we generated the truncation mutants of PDK1 (Fig. 1E) and performed the GST pull-down assay. HA-ULK1 was bound to GST-PDK1 WT, KL and C but not $\mathrm{PH}$ (Fig. 1F), suggesting that the linker region of PDK1 interacts with ULK1.

\section{ULK1 phosphorylates Ser389 of PDK1 linker region}

To identify the region of PDK1 that is phosphorylated by ULK1, GST-PDK1 kinase domain, C, and PH were overexpressed with HA-ULK1. Only GST-PDK1 C showed the slowmigrating species in SDS-PAGE (Fig. 2A), indicating that ULK1 may phosphorylate the linker region of PDK1. Next, the in vitro kinase assay using the bacterially purified GST-PDK1 linker protein as a substrate showed that ULK1 WT directly phosphorylated the substrate protein in vitro, while ULK1 KI form was not (Fig. 2B). As ULK1 is an evolutionarily conserved ser/thr kinase from yeast to humans, we speculated that the phosphorylation sites of PDK1 may be conserved. Among evolutionarily conserved serine/threonine residues, several sites were mutated to alanine. However, none of the mutants showed the fast-migrating bands compared with wild-type PDK1 (data not shown). To obtain the phosphorylated PDK1 proteins for mass spectrometry analysis, HEK293T cells were transfected with ULK1 and GST-PDK1 linker. The eluted proteins were subjected to mass spectrometry analysis (Fig. 2C). Finally, Ser389 was identified as the phosphorylation site (Fig. 2D).

To confirm this result, Ser389 residues were mutated to alanine (S389A) and aspartate (S389D), and these mutant proteins were expressed with HA-ULK1. PDK1 WT showed band shift in the presence of ULK1, whereas PDK1 S389A and S389D did not (Fig. 3A). In order to investigate the characteristics of PDK1 phosphorylation at Ser389, we generated phospho-specific antibody that recognizes phosphorylated
Ser389 of PDK1 (pS389 PDK1 antibody). HEK293T cells transfected with PDK1 alone or together with ULK1 were lysed and PDK1 proteins were immunoprecipitated using anti-Myc antibody. In line with the above data, pS389 antibody specifically recognized the PDK1 proteins that are co-expressed with ULK1 (Fig. 3B). Additionally, overexpression of ULK1 WT increased the Ser389 phosphorylation of endogenous PDK1, while ULK1 KI did not (Fig. 3C). Next, we tested whether the activation of ULK1 affected the phosphorylation of PDK1. We used oligomycin A, which is known to induce energy depri-
A

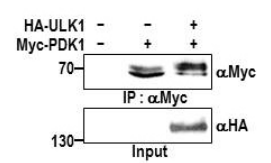

C

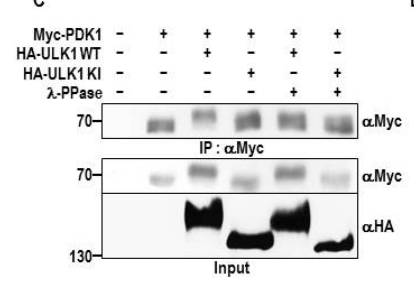

$E$

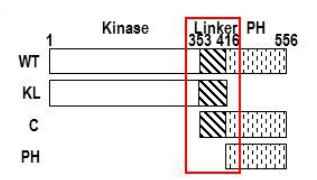

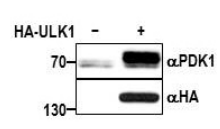

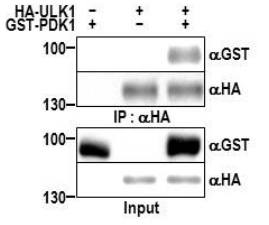

$\mathrm{F}$

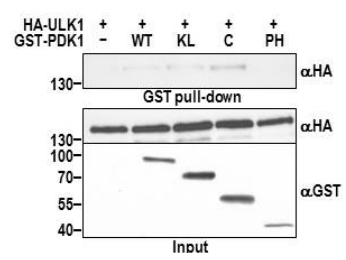

Fig. 1. ULK1 interacts with PDK1 and induces phosphorylation. (A) HEK293T cells were transfected with HA-ULK1 and Myc-PDK1 constructs. After $48 \mathrm{~h}$ of transfection, the cells were lysed, and the lysates were subjected to immunoprecipitation using anti-Myc antibody. The immune complexes were washed and the immunoblots for PDK1 were performed as described in Materials and Methods (upper panel). The same cell lysates were used for anti-HA immunoblot (lower panel). (B) HA-ULK1 was expressed in HEK293T cells. Whole cell lysate was analyzed by immunoblotting for the indicated proteins. (C) HEK293T cells transfected with Myc-PDK1 and HA-ULK1 wild-type (WT) or kinase-inactive (KI) mutant were lysed and subject to immunoprecipitation with anti-Myc antibody. The immune complexes were incubated with $\lambda$ phosphatase, washed, and subjected to anti-Myc immunoblot as described in Materials and Methods (top panel). Anti-Myc and anti-HA immunoblots were conducted using the same cell lysates (middle and bottom panels, respectively). (D) HA-ULK1 and GST-PDK1 were expressed in the HEK293T cells. The cell lysates were subjected to immunoprecipitation using anti-HA antibody. Immunoblots were obtained with the indicated antibodies. (E) The scheme of PDK1 truncation mutants. (F) HEK293T cells transfected with HA-ULK1 and GST-PDK1 $\mathrm{WT}$, kinase and linker $(\mathrm{KL}), \mathrm{C}$-terminal $(\mathrm{C})$ or $\mathrm{PH}$ domain $(\mathrm{PH})$ constructs were lysed, and the lysates were subjected to co-immunoprecipitation. The results shown are representative of three independent experiments. 


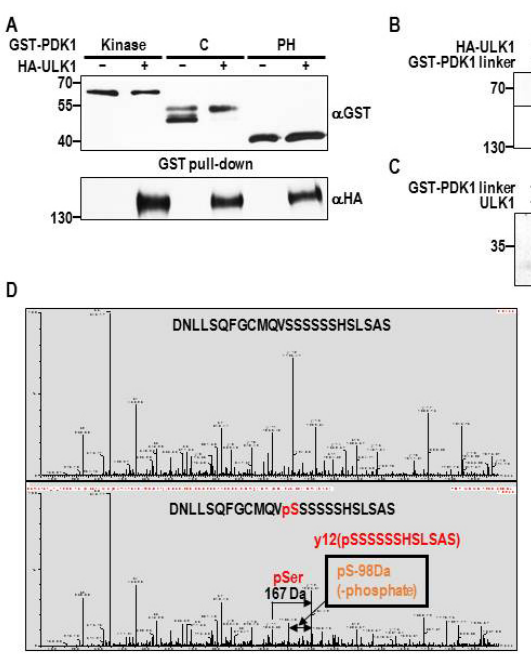

Fig. 2. ULK1 phosphorylates Ser389 of PDK1 linker region. (A) HA-ULK1 proteins were co-expressed with GST-PDK1 Kinase, C or $\mathrm{PH}$ proteins in HEK293T cells. The cell lysates were cleared and incubated with glutathione beads as described in Materials and Methods. Western blots for the precipitated proteins were obtained using anti-GST antibody (top panel). The anti-HA immunoblot (bottom panel) was also conducted using the same whole cell lysates. (B) HEK293T cells transfected with HA-ULK1 WT or KI were lysed and ULK1 assays were performed using GST-PDK1 linker protein as a substrate as described in Materials and Methods. The phosphorylated proteins were visualized by autoradiography (top panel). ULK1 expression was detected by the immunoblot obtained from the same whole cell lysates (bottom panel). The above results shown are representative of three independent experiments. (C) HEK293T cells were transfected with GST-PDK1 linker and HA-ULK1 WT. After $48 \mathrm{~h}$ of transfection, PDK1 linker proteins were pulled down and eluted as described in Materials and Methods. The fractions of eluted proteins were subjected to SDS-PAGE and the gel was stained by Coomassie R250 solution. (D) Phosphorylated proteins from $(C)$ were analyzed by mass spectrometry as described in Materials and Methods.

vation, and rapamycin, which inhibits mTOR and induces subsequent increase in ULK1 activity. Oligomycin A treatment induced Ser389 phosphorylation of PDK1 at $4 \mathrm{~h}$ (Fig. 3D). Rapamycin also elicited PDK1 phosphorylation (Fig. 3E). Furthermore, the addition of ULK1 inhibitor decreased PDK1 phosphorylation induced by oligomycin A (Fig. 3F). Taken together, these results confirmed that ULK1 phosphorylates PDK1 at Ser389.

Phosphorylation at Ser389 may affect downstream signaling To elucidate the signaling effect of Ser389 phosphorylation of PDK1, we used the PDK1 knockout cell line. Stable cell lines expressing PDK1 wild type or S389A mutant in PDK1 knockout cell line were established (Fig. 4A). First, the in vitro kinase assay was carried out to measure the activity of PDK1. It was intriguing that the activity of PDK1 S389A is very similar to wild type in vitro (Fig. 4A). When the phosphorylation status

\section{A}

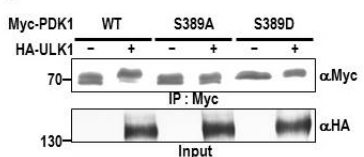

Byc-PDK1 $= \pm+$

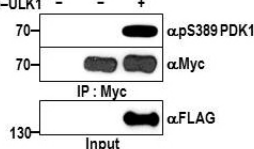

C

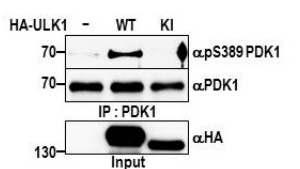

$\begin{array}{llllll}\text { Oligomycin A } & 0 & 2 & 4 & 8 & \text { (hr) }\end{array}$
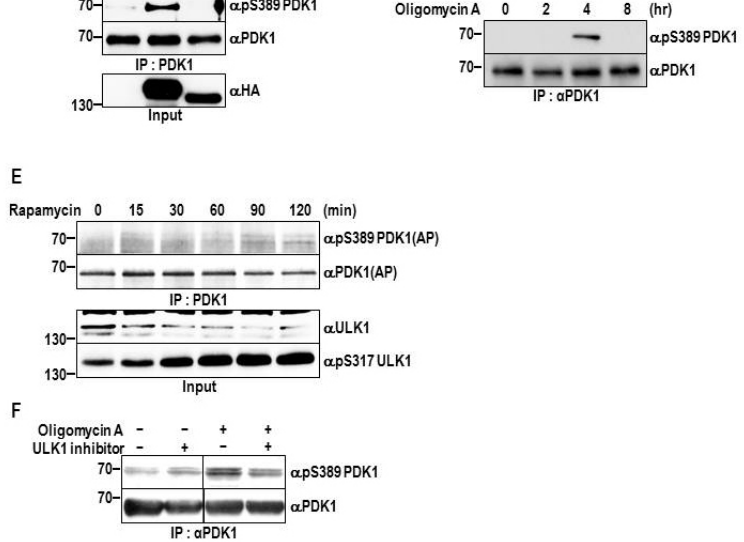

Fig. 3. ULK1 phosphorylates Ser389 of PDK1. (A) HEK293T cells were transfected with the indicated constructs. After $48 \mathrm{~h}$ of transfection, the cells were lysed and the lysates were subjected to immunoprecipitation using anti-Myc antibody (top panel). ULK1 immunoblot was performed using the same cell lysates (bottom panel). (B) Myc-PDK1 and Flag-ULK1 were overexpressed in the HEK293T cells. Immunoprecipitated Myc-PDK1 proteins were probed with an anti-pS389 PDK1 antibody (top panel). (C) HA-ULK1 WT and KI were overexpressed in the HEK293T cells and endogenous PDK1 proteins were immunoprecipitated using the antiPDK1 antibody (top and middle panel). ULK1 expression was detected in the same cell lysates (bottom panel). (D) After the addition of $5 \mu \mathrm{M}$ Oligomycin A to HEK293T cells at the indicated time points, the cells were lysed for immunoprecipitation of PDK1. The immune complexes were subjected to western blot using anti-pS389 and anti-PDK1 antibodies (top and bottom panel, respectively) (E) HEK293T cells were treated with $1 \mu \mathrm{M}$ rapamycin at the indicated time points. Immunoprecipitated PDK1 proteins were probed with anti-pS389 and anti-PDK1 antibodies (top and upper middle panels, respectively). AP indicates alkaline phosphatase blotting. (F) HEK293T cells were treated with ULK1 inhibitor (1 $\mu \mathrm{M}, \mathrm{SBl}-0206965)$ for $20 \mathrm{~h}$, followed by addition of oligomycin A $(5 \mu \mathrm{M})$ or DMSO for $4 \mathrm{~h}$. Immunoprecipitated proteins using PDK1 antibody were analyzed via immunoblotting using the indicated antibodies. The above results shown are representative of three independent experiments.

of the downstream targets of PDK1 were investigated, however, S389A mutant showed relatively weak activity toward Akt and p70S6k compared with wild type (Fig. 4B). These data suggested that Ser389 phosphorylation of PDK1 by ULK1 is necessary for the expression of upstream signals. 


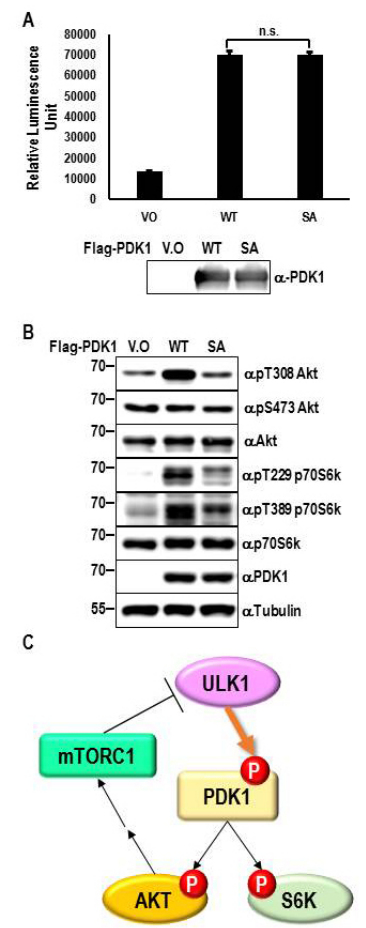

Fig. 4. Phosphorylation of PDK1 Ser389 regulates substrate phosphorylation. (A) Flag-PDK1 WT and S389A proteins were purified from HAP1 stable cells as described in Materials and Methods. PDK1 kinase activity was measured using ADP-Glo ${ }^{{ }^{M}}$ PDK1 kinase assay kit according to the manufacturer's instructions. The data represent means \pm SD of three experiments. Statistical analysis was performed using Student's t-test, and P-value $<0.05$ was considered significant; however, the calculated $P$ value was 0.69. n.s.: not significant (B) HAP1 PDK1 knockout cells stably expressing PDK1 WT and S389A mutant were lysed and the whole cell lysates were analyzed by immunoblotting using the indicated antibodies. (C) Negative feedback loop model between PDK1 and ULK1.

\section{DISCUSSION}

Since PDK1 is known as a master kinase belonging to the AGC kinase family and is a nearly constitutively active enzyme, its activity depends on the readiness of substrates for phosphorylation by PDK1. For example, the phosphorylation of p70s6k by PDK1 depends on the phosphorylation at a C-terminal Ser/Thr residue located in the hydrophobic motif (27). This phosphorylation facilitates binding of PDK1 to this kinase via a specific substrate-docking site termed the 'PIF pocket' (28), whereas the activation of Akt by PDK1 is independent of phosphorylation at the hydrophobic motif $(27,28)$.

Other groups investigated the regulation of PDK1 itself. For instance, sphingosine increased PDK1 phosphorylation over 25-fold (29). In addition, PDK1 autophosphorylated Ser241 residue, and this phosphorylation is required for Akt activation
(11). Serine residues in the linker region (Ser393, 396, and 410) were phosphorylated in HEK293 cells. However, this phosphorylation was not required for downstream signaling. Furthermore, insulin promoted the phosphorylation of PDK1 at Tyr9 and 373/376 in the plasma membrane (30). Although Tyr$373 / 376$ residues were located in the linker region, none of these was involved in PDK1 activity.

To our knowledge, this work is the first report demonstrating the phosphorylation of Ser389 in PDK1. Based on previous reports, a few hypotheses can be proposed: First, since Ser389 is located in the linker region between kinase and $\mathrm{PH}$ domains of PDK1, phosphorylation at this residue might induce conformational changes in PDK1 protein, leading to altered substrate recognition of the kinase, which is supported by the results suggesting that PDK1 kinase activity was not altered in S389A mutant (Fig. 4A). Further, the phosphorylation by ULK1 may impede inhibitory homodimerization of PDK1 similar to the phosphorylation in the $\mathrm{PH}$ domain $(15,16,31)$. Finally, there is a possibility that the subcellular localization of PDK1 may be modulated by the phosphorylation at Ser389.

ULK1 protein complex is directly regulated by mTORC1 as mentioned above. In addition, the feedback mechanisms of ULK1 to mTORC1 have been investigated. Two independent groups demonstrated that ULK1 phosphorylated raptor, a component of mTORC1 complex, and subsequently inhibited mTORC1 activity $(24,25)$, which was consistent with previous data showing that ULK1 blocked p70S6k (22). However, another report suggested that ULK1 phosphorylated all three subunits of AMP-activated kinase (AMPK) resulting in its inhibition (23). However, the role of these phosphorylations in mTORC1 signaling is unknown. It is well known that the inhibition of AMPK activity generally leads to activation of mTORC1 via TSC $1 / 2$ complex and raptor (32), in line with our data suggesting that PDK1 phosphorylation by ULK1 might be necessary for the activation of downstream targets of PDK1 (Fig. 4B). Thus, ULK1 might activate or inhibit mTORC1, which might appear inherently contradictory. However, specific conditions might determine the direction of feedback. Further studies are needed to elucidate the mechanism underlying this novel feedback loop.

In conclusion, our study suggests that Ser389 phosphorylation of PDK1 regulates its signaling function and the existence of a novel negative feedback loop between PDK1 and ULK1/ autophagy pathway.

\section{MATERIALS AND METHODS}

Cell culture, transfection, and establishing stable cell lines HEK293T cells were cultured in Dulbecco's Modified Eagle Medium (WELGENE, Daegu, Republic of Korea) supplemented with $10 \%$ fetal bovine serum (FBS) (ThermoFisher, Waltham, MA, USA) and antimycotics (ThermoFisher). Human PDK1 knockout HAP1 cells were purchased from Horizon and cultured in Iscove's Modified Dulbecco's Medium (WELGENE) 
supplemented with $10 \%$ FBS and antimycotics. Cells were transfected using X-tremeGENE ${ }^{\mathrm{TM}}$ (Roche, Basel, Switzerland) according to the manufacturer's protocol. PDK1 knockout HAP1 cells were infected with lentivirus particles harboring PDK1 wild-type or S389A constructs and selected by adding puromycin $(1 \mu \mathrm{g} / \mathrm{mL})$ for 7 to 10 days. Western blot confirmed PDK1 protein expression by the selected cells.

\section{Antibodies and reagents}

Anti-Flag M2 (F1804), anti-GST (G7781), anti-Tubulin (T6074), Oligomycin A (75351) and Rapamycin (R8781) were obtained from Merck (St. Louis, MO, USA). Anti-Myc (sc-40), anti-HA (sc-805) and anti-GAPDH (sc-47724) were purchased from Santa Cruz Biotechnology (Dallas, TX, USA). Anti-Myc (2278S), anti-Phospho-ULK1 Ser317 (6887S), anti-ULK1 (8054S), antiPhospho-Akt Ser473 (4060S), anti-Phospho-Akt Thr308 (4056S), anti-Akt (4691S), anti-Phospho-p70S6k Thr389 (9234S), antip70S6k (2708S) and anti-PDK1 (3062S) antibodies were obtained from Cell Signaling Technology (Danvers, MA, USA). Anti-Phospho-p70Sk Thr229 (MAB8964) was purchased from R\&D systems (Minneapolis, MN, USA). The phospho-specific antibody recognizing PDK1 phosphorylated at Ser389 was raised in rabbits against the peptide CMQVSSSSSSSHS (corresponding to residues 385-396 of human PDK1) in which the underlined residue is phosphoserine (Peptron, Daejeon, Republic of Korea). The antibodies were affinity-purified on activated-Sepharose (ThermoFisher) covalently coupled to the phosphorylated peptide and then passed through a column of $\mathrm{CH}$-Sepharose coupled to the non-phosphorylated peptide. Antibodies that did not bind to the latter column were selected. ULK1 inhibitor, SBI-0206965 (S7885) were obtained from Selleckchem (Houston, TX, USA). Anti-HA Magnetic Beads (88837), Glutathione Magnetic Agarose Beads (78601), Anti-c-Myc Magnetic Beads (88843), Anti-DYKDDDDK Magnetic Agarose (A36798) and Dynabeads ${ }^{\mathrm{TM}}$ Protein G for Immunoprecipitation (10004D) were purchased from ThermoFisher and used for immunoprecipitation.

\section{Preparation of cell lysates, immunoprecipitation, and in vitro kinase assay}

Stimulation was terminated by washing cells with ice-cold PBS (10 mM Na $2 \mathrm{HPO}_{4}, 1.76 \mathrm{mM} \mathrm{KH} \mathrm{PO}_{4}, 137 \mathrm{mM} \mathrm{NaCl}, 2.7 \mathrm{mM}$ $\mathrm{KCl})$. Cell lysates were prepared in Buffer A $(20 \mathrm{mM}$ Tris- $\mathrm{HCl}$ (pH 7.5), 0.1\% Triton X-100, 1 mM EDTA, 5 mM EGTA, $10 \mathrm{mM}$ $\mathrm{MgCl}_{2}, 50 \mathrm{mM} \beta$-glycerophosphate, $1 \mathrm{mM}$ sodium orthovanadate, $1 \mathrm{mM}$ dithiothreitol, $1 \mathrm{X}$ protease inhibitor cocktail (Roche)).

To perform ULK1 kinase assay, the protein-bead complexes were washed twice with Buffer $A$, then twice with Buffer $A$ containing $500 \mathrm{mM} \mathrm{NaCl}$. Finally, the complexes of ULK1 were washed with Buffer B containing $20 \mathrm{mM}$ HEPES ( $\mathrm{pH}$ 7.2), $10 \mathrm{mM} \mathrm{MgCl} 2,0.1 \mathrm{mg} / \mathrm{ml} \mathrm{BSA}$, and $3 \mathrm{mM} \beta$-mercaptoethanol. ULK1 activities were assayed in a reaction mixture consisting of 1X Buffer B, $1 \mu \mathrm{g}$ GST-PDK1 Linker protein, $20 \mu \mathrm{M}$ ATP, and $10 \mu \mathrm{Ci}$ of $\left[\gamma^{32} \mathrm{P}\right] \mathrm{ATP}$ at $30^{\circ} \mathrm{C}$ for $15 \mathrm{~min}$.

PDK1 KO HAP1 cells stably expressing Flag-PDK1 wild-type and S389A mutant cells were harvested and lysed with Buffer A containing $1 \mathrm{X}$ phosphatase inhibitor (Roche). Lysates were immunoprecipitated with Anti-DYKDDDDK magnetic beads. The protein-bead complexes were washed twice with Buffer A containing $500 \mathrm{mM} \mathrm{NaCl}$, twice with Buffer A containing $250 \mathrm{mM}$ $\mathrm{NaCl}$, and finally once with Buffer $\mathrm{A}$, and then eluted using $3 \mathrm{X}$ flag peptide. Purified PDK1 enzymes were used for ADP-Glo ${ }^{\text {TM }}$ PDK1 kinase assay (Promega, Madison, WI, USA) according to the manufacturer's instructions.

\section{Mass spectrometry}

HEK293T cells were transfected with pEBG-PDK1 linker and after $48 \mathrm{~h}$, the cells were lysed with Buffer A. The overexpressed proteins were pulled down by glutathione Sepharose 4B (GE Healthcare, Chicago, IL, USA). The protein-bead complexes were washed twice with Buffer A, twice with Buffer A containing $500 \mathrm{mM} \mathrm{NaCl}$, and finally once with ST buffer containing $50 \mathrm{mM}$ Tris- $\mathrm{HCl} \mathrm{pH} 7.4$ and $150 \mathrm{mM} \mathrm{NaCl}$. The proteins were eluted with Buffer $\mathrm{C}$ containing $30 \mathrm{mM}$ glutathione and PBS. Eluted proteins were concentrated by Microcon (Merck) and analyzed using 2D electrophoresis, MALDITOF, and Q-TOF by In2Gen.

\section{ACKNOWLEDGEMENTS}

This work was supported by a grant (NRF-2019M3E5D4069882) from the National Research Foundation, Ministry of Science and ICT, and another grant from KRIBB initiative program.

\section{CONFLICTS OF INTEREST}

The authors have no conflicting interests.

\section{REFERENCES}

1. Rameh LE and Cantley LC (1999) The role of phosphoinositide 3-kinase lipid products in cell function. J Biol Chem 274, 8347-8350

2. Anderson KE, Coadwell J, Stephens LR and Hawkins PT (1998) Translocation of PDK-1 to the plasma membrane is important in allowing PDK-1 to activate protein kinase B. Curr Biol 8, 684-691

3. Alessi DR, James SR, Downes CP et al (1997) Characterization of a 3-phosphoinositide-dependent protein kinase which phosphorylates and activates protein kinase Balpha. Curr Biol 7, 261-269

4. Stephens L, Anderson K, Stokoe D et al (1998) Protein kinase B kinases that mediate phosphatidylinositol 3,4,5-trisphosphate-dependent activation of protein kinase B. Science $279,710-714$

5. Pullen N, Dennis PB, Andjelkovic M et al (1998) Phosphorylation and activation of p70s6k by PDK1. Science $279,707-710$ 
6. Lang F and Cohen P (2001) Regulation and physiological roles of serum- and glucocorticoid-induced protein kinase isoforms. Sci Stke 2001, re17

7. Jensen CJ, Buch MB, Krag TO, Hemmings BA, Gammeltoft $S$ and Frodin $M$ (1999) 90-kDa ribosomal S6 kinase is phosphorylated and activated by 3-phosphoinositide-dependent protein kinase-1. J Biol Chem 274, 27168-27176

8. Dutil EM, Toker A and Newton AC (1998) Regulation of conventional protein kinase $\mathrm{C}$ isozymes by phosphoinositidedependent kinase 1 (PDK-1). Curr Biol 8, 1366-1375

9. Le Good JA, Ziegler WH, Parekh DB, Alessi DR, Cohen P and Parker PJ (1998) Protein kinase C isotypes controlled by phosphoinositide 3-kinase through the protein kinase PDK1. Science 281, 2042-2045

10. Alessi DR, Deak M, Casamayor A et al (1997) 3-Phosphoinositide-dependent protein kinase-1 (PDK1): structural and functional homology with the Drosophila DSTPK61 kinase. Curr Biol 7, 776-789

11. Casamayor A, Morrice NA and Alessi DR (1999) Phosphorylation of Ser-241 is essential for the activity of 3-phosphoinositide-dependent protein kinase-1: identification of five sites of phosphorylation in vivo. Biochem J 342 ( Pt 2), 287-292

12. Gao X, Lowry PR, Zhou X et al (2011) PI3K/Akt signaling requires spatial compartmentalization in plasma membrane microdomains. Proc Natl Acad Sci U S A 108, 14509-14514

13. Lim MA, Kikani CK, Wick MJ and Dong LQ (2003) Nuclear translocation of 3'-phosphoinositide-dependent protein kinase 1 (PDK-1): a potential regulatory mechanism for PDK-1 function. Proc Natl Acad Sci U S A 100, 14006-14011

14. Kim S, Jee K, Kim D, Koh H and Chung J (2001) Cyclic AMP inhibits Akt activity by blocking the membrane localization of PDK1. J Biol Chem 276, 12864-12870

15. Masters TA, Calleja V, Armoogum DA et al (2010) Regulation of 3-phosphoinositide-dependent protein kinase 1 activity by homodimerization in live cells. Sci Signal 3, ra78

16. Wick MJ, Ramos FJ, Chen H, Quon MJ, Dong LQ and Liu F (2003) Mouse 3-phosphoinositide-dependent protein kinase-1 undergoes dimerization and trans-phosphorylation in the activation loop. J Biol Chem 278, 42913-42919

17. Kamada Y, Funakoshi T, Shintani T, Nagano K, Ohsumi M and Ohsumi Y (2000) Tor-mediated induction of autophagy via an Apg1 protein kinase complex. J Cell Biol 150, 1507-1513

18. Kuroyanagi $\mathrm{H}$, Yan J, Seki $\mathrm{N}$ et al (1998) Human ULK1, a novel serine/threonine kinase related to UNC-51 kinase of Caenorhabditis elegans: cDNA cloning, expression, and chromosomal assignment. Genomics 51, 76-85

19. Yan J, Kuroyanagi $H$, Kuroiwa $A$ et al (1998) Identification of mouse ULK1, a novel protein kinase structurally related to C. elegans UNC-51. Biochem Biophys Res Commun 246, 222-227

20. Matsuura A, Tsukada M, Wada Y and Ohsumi Y (1997) Apg1p, a novel protein kinase required for the autophagic process in Saccharomyces cerevisiae. Gene 192, 245-250

21. Kamada Y, Yoshino K, Kondo C et al (2010) Tor directly controls the Atg1 kinase complex to regulate autophagy. Mol Cell Biol 30, 1049-1058

22. Lee SB, Kim S, Lee J et al (2007) ATG1, an autophagy regulator, inhibits cell growth by negatively regulating $\mathrm{S} 6$ kinase. EMBO Rep 8, 360-365

23. Loffler AS, Alers S, Dieterle AM et al (2011) Ulk1-mediated phosphorylation of AMPK constitutes a negative regulatory feedback loop. Autophagy 7, 696-706

24. Dunlop EA, Hunt DK, Acosta-Jaquez HA, Fingar DC and Tee AR (2011) ULK1 inhibits mTORC1 signaling, promotes multisite Raptor phosphorylation and hinders substrate binding. Autophagy 7, 737-747

25. Jung $\mathrm{CH}$, Seo M, Otto NM and Kim DH (2011) ULK1 inhibits the kinase activity of mTORC1 and cell proliferation. Autophagy 7, 1212-1221

26. Gao X and Harris TK (2006) Steady-state kinetic mechanism of PDK1. J Biol Chem 281, 21670-21681

27. Collins BJ, Deak M, Arthur JS, Armit LJ and Alessi DR (2003) In vivo role of the PIF-binding docking site of PDK1 defined by knock-in mutation. EMBO J 22, 42024211

28. Biondi RM, Kieloch A, Currie RA, Deak M and Alessi DR (2001) The PIF-binding pocket in PDK1 is essential for activation of S6K and SGK, but not PKB. EMBO J 20, 4380-4390

29. King CC, Zenke FT, Dawson PE et al (2000) Sphingosine is a novel activator of 3-phosphoinositide-dependent kinase 1. J Biol Chem 275, 18108-18113

30. Park J, Hill MM, Hess D, Brazil DP, Hofsteenge J and Hemmings BA (2001) Identification of tyrosine phosphorylation sites on 3-phosphoinositide-dependent protein kinase- 1 and their role in regulating kinase activity. J Biol Chem 276, 37459-37471

31. Ziemba BP, Pilling C, Calleja V, Larijani B and Falke JJ (2013) The PH domain of phosphoinositide-dependent kinase-1 exhibits a novel, phospho-regulated monomer-dimer equilibrium with important implications for kinase domain activation: single-molecule and ensemble studies. Biochemistry 52, 4820-4829

32. Saxton RA and Sabatini DM (2017) mTOR Signaling in Growth, Metabolism, and Disease. Cell 168, 960-976 